\title{
THE SHAPE OF LEVEL CURVES ${ }^{1}$
}

GEORGE PIRANIAN

Let $P(z)=\prod_{m=1}^{n}\left(z-z_{m}\right)^{k_{m}}$, and let the positive number $c$ be small enough so that the set

$$
E(c)=\{z:|P(z)|<c\}
$$

has $n$ components. H. Grunsky [1, Problem 16] raised the question whether the components of $E(c)$ are then necessarily convex. Ch. Pommerenke showed that in the case where $P$ has exactly two zeros, one simple, the other of sufficiently high multiplicity, the answer is negative [3, Theorem 14]. Recently, A. W. Goodman [2] exhibited a quartic such that for some values $c$ the set $E(c)$ has four components, one not convex. In $\$ 1$, I show that each third-order lemniscate with a double point has a loop that is not convex.

In [2], Goodman conjectured that if $E(c)$ has $n$ components, then each component is starlike with respect to the zero that it contains. I prove that the conjecture is false for certain polynomials of high degree $(\S 2)$.

\section{Convexity.}

THEOREM 1. Let $f$ be holomorphic at $z_{0}$, and in the Taylor expansion $f(z)=\sum a_{k}\left(z-z_{0}\right)^{k}$, let the first three nonzero coefficients be $a_{0}, a_{m}, a_{n}$, with $m \geqq 2$. If $n>2 m$, then all components of the set

$$
E=\left\{z:|f(z)|<\left|f\left(z_{0}\right)\right|\right\}
$$

are convex in some neighborhood of $z_{0}$; if $n<2 m$, then at least one component is not convex near $z_{0}$.

COROLlary. If $f$ is a cubic with two distinct critical points $z_{1}$ and $z_{2}$, and if $f\left(z_{1}\right) \neq 0$, then one component of the set

$$
E=\left\{z:|f(z)|<\left|f\left(z_{1}\right)\right|\right\}
$$

is not convex.

Proof of the theorem. After we multiply $f(z)$ by $1 / f\left(z_{0}\right)$ and move the origin to $z_{0}$, our function has the Taylor expansion

$$
f(z)=1+a_{m} z^{m}+a_{n} z^{n}+\cdots ;
$$

Received by the editors May 23, 1966.

1 This note was written with support from the National Science Foundation. 
we then rotate the z-plane through an angle chosen so that the constant $a_{m}$ in the expansion becomes an imaginary number $i \alpha$. Since the tangent lines to the lemniscate $|f(z)|=|f(0)|$ at the origin are the lines on which $|f(z)|$ is most nearly constant, the tangent rays are then the half-lines

$$
z=r e^{\pi i j / m} \quad(j=0,1, \cdots, 2 m-1) .
$$

With the notation $a_{n} e^{\pi i j n / m}=A_{j}+i B_{j}(j=1, \cdots, 2 m-1)$, we can estimate the quantity $|f(z)|$ on the $j$ th tangent ray by the formula

$$
\left|f\left(r e^{\pi i j / m}\right)\right|=\left|1+i \alpha r^{m}+\left(A_{j}+i B_{j}\right) r^{n}+O\left(r^{n+1}\right)\right| .
$$

Suppose now that $n>2 m$. Then the relation

$$
\left|f\left(r e^{\pi i j / m}\right)\right|^{2}=1+\alpha^{2} r^{2 m}+O\left(r^{2 m+1}\right)
$$

holds for each index $j$. It implies that on each of the tangent lines, $|f(z)|>1$ throughout some punctured neighborhood of the origin. This means that near the origin the tangent lines lie outside of the set $E(1)$, and since $f$ is holomorphic, it follows that each component of $E(1)$ is convex near the origin.

Suppose next that $n<2 m$. Then

$$
\left|f\left(r e^{\pi i j / m}\right)\right|^{2}=1+2 A_{j} r^{n}+O\left(r^{n+1}\right),
$$

and since $A_{j}$ is negative for at least $m-1$ indices $j$, at least $m-1$ of the tangent rays meet the set $E(1)$ near the origin.

Suppose that $R$ is a tangent ray that meets $E(1)$ near the origin; let $z_{1}$ and $z_{2}$ be two points on opposite sides of $R$ and near enough to $R$ so that they lie in the component of $E(1)$ to which $R$ is tangent. Since the radius vector of one of the two points lies outside of the component, in some neighborhood of the origin, the component is not convex near the origin. This completes the proof of Theorem 1.

\section{Components that are not starlike.}

THEOREM 2. If the natural number $k$ is large enough, then there exists a positive number a such that the set

$$
E(a)=\left\{z:\left|\left(z^{3}-1\right)^{k}\left(z^{3}-i a\right)\right|<a\right\}
$$

has six components, three of them not starlike relative to the zero of $f$ that they contain.

Let $f$ denote the polynomial in the statement of the theorem. The principal idea in the proof is to place the three simple zeros on the rays

$$
z=r e^{i \pi(4 j+1) / 6} \quad(j=0,1,2)
$$


at the appropriate distance from the origin so that the level curve

$$
\Gamma(a)=\{z:|f(z)|=a\}
$$

through the origin has three double points. We shall show that for large values of $k$, this brings the simple zeros and the corresponding double points close to the origin. We shall further show that the line segment from a double point to the corresponding multiple zero then lies in a direction in which $|f(z)|$ increases, near the double point.

It remains only to supply the details of the argument in the preceding paragraph. We note that

$$
f^{\prime}(z)=3 z^{2}\left(z^{3}-1\right)^{k-1}\left\{(k+1) z^{3}-(i k a+1)\right\},
$$

and that therefore the level curve through the origin has three double points if we choose the value $a$ so that

$$
|f(z)|=a \quad \text { when } \quad z^{3}=\frac{i k a+1}{k+1} .
$$

To solve the equation

$$
\left|1-\frac{i k a+1}{k+1}\right|^{k}\left|\frac{i k a+1}{k+1}-i a\right|=a \quad(a>0),
$$

we make elementary rearrangements in the left member and multiply both sides by $k(1+1 / k)^{k+1}$. We thus obtain the equation

$$
|1-i a|^{k+1}=(1+1 / k)^{k+1} k a,
$$

and we rewrite this in the form

$$
\begin{aligned}
1+a^{2} & =(1+1 / k)^{2} \exp \frac{2 \log k a}{k+1} \\
& =(1+1 / k)^{2}\left\{1+\frac{2 \log k a}{k+1}+\frac{1}{2 !}\left(\frac{2 \log k a}{k+1}\right)^{2}+\cdots\right\} .
\end{aligned}
$$

For all large values of $k$, the equation has a solution between $a=k^{-1 / 2}$ and $a=k^{-1 / 3}$. Therefore the three double points of the curve $\Gamma(a)$ approach the origin, as $k$ becomes large.

To iden tify the directions of most rapid change of $|f(z)|$ at a double point $z_{0}$ of $\Gamma(a)$, we note that

$$
f^{\prime \prime}\left(z_{0}\right)=9(k+1) z_{0}^{4}\left(z_{0}^{3}-1\right)^{k-1}
$$

and

$$
f(z)=f\left(z_{0}\right)+(1 / 2) f^{\prime \prime}\left(z_{0}\right)\left(z-z_{0}\right)^{2}+O\left(\left|z-z_{0}\right|^{3}\right) .
$$


The change along a fixed direction is most rapid if the first two terms on the right have the same argument, and since

$$
\frac{f^{\prime \prime}\left(z_{0}\right)}{f\left(z_{0}\right)}=\frac{9(k+1) z_{0}^{4}}{\left(z_{0}^{3}-1\right)\left(z_{0}^{3}-i a\right)}=\frac{9(k+1)^{5 / 3}(i k a+1)^{4 / 3}}{(i k a-k)(1-i a)},
$$

we see that

$$
\arg f^{\prime \prime}\left(z_{0}\right)=\arg f\left(z_{0}\right)+\frac{4 \pi}{3 \cdot 2}-\pi+o(1)=\arg f\left(z_{0}\right)-\pi / 3+o(1) .
$$

It follows that in one of the directions of most rapid growth, $\arg \left(z-z_{0}\right)$ $\sim \pi / 6$. This implies that the inequality $|f(z)|>\left|f\left(z_{0}\right)\right|$ holds throughout some domain

$$
\left\{z:\left|z-z_{0}\right|<\epsilon, \frac{\pi}{6}-\left(\frac{\pi}{4}-\epsilon\right)<\arg \left(z-z_{0}\right)<\frac{\pi}{6}+\left(\frac{\pi}{4}-\epsilon\right)\right\} .
$$

It follows that the component containing the point 1 is not starlike at 1 , and the proof is complete.

REMARK. A rough sketch shows that in our example each component of $E(a)$ is starlike relative to one of its points. However, it is clear how our construction can be modified so that in addition to the corner at the origin, the boundary of a component of $E(a)$ has two corners, situated so that from each point of the component one of them is invisible. Also, it is possible to construct a polynomial $f$ such that each component of $E(|f(0)|)$ contains exactly one zero (generally of high multiplicity), and such that one component spirals about a critical point of $f$ a prescribed number of times.

\section{REFERENCES}

1. P. Erdös, F. Herzog and G. Piranian, Metric properties of polynomials, J. Analyse Math. 6 (1958), 125-148.

2. Ch. Pommerenke, On metric properties of complex polynomials, Michigan Math. J. 8 (1961), 97-115.

3. A. W. Goodman, On the convexity of the level curves of a polynomial, Proc. Amer. Math. Soc. 17 (1966), 358-361.

The University of Michigan 